\title{
Role of Neurokinin 3 Receptor Signaling in Oral Squamous Cell Carcinoma
}

\author{
KYOICHI OBATA ${ }^{1}$, TSUYOSHI SHIMO ${ }^{1}$, TATSUO OKUI ${ }^{1}$, KENICHI MATSUMOTO $^{1}$, \\ HIROYUKI TAKADA ${ }^{1}$, KIYOFUMI TAKABATAKE ${ }^{2}$, YUKI KUNISADA ${ }^{1}$, SOICHIRO IBARAGI ${ }^{1}$, \\ NORIE YOSHIOKA ${ }^{1}$, KOJI KISHIMOTO ${ }^{1}$, HITOSHI NAGATSUKA ${ }^{2}$ and AKIRA SASAKI ${ }^{1}$ \\ ${ }^{1}$ Department of Oral and Maxillofacial Surgery, Okayama University Graduate School of Medicine, \\ Dentistry, and Pharmaceutical Sciences, Okayama, Japan; \\ ${ }^{2}$ Department of Oral Pathology and Medicine, Okayama University Graduate School of Medicine, \\ Dentistry, and Pharmaceutical Sciences, Okayama, Japan
}

\begin{abstract}
Background/Aim: The neurokinin 3 receptor (NK$3 R$ ) is differentially expressed in the central nervous system including cases of human oral squamous cell carcinoma. However, the role of $N K-3 R$ signaling in oral squamous cell carcinoma is not well known. Materials and Methods: NK-3R expression in surgically resected oral squamous cell carcinoma was examined immunohistochemically and the strength of the expression was quantified. We evaluated the function of $N K-3 R$ signaling using $N K-3 R$ antagonist in human oral squamous cell carcinoma bone invasion mouse model. Results: $N K-3 R$ was significantly expressed in tumor cells that had invaded the bone matrix compared to the oral side tumor cells. SB222200, a selective antagonist of $N K-3 R$, significantly suppressed the radiographic osteolytic lesion and tumorigenesis. Conclusion: $N K-3 R$ signaling is a potential target for the treatment of oral squamous cell carcinoma in cases of bone destruction.
\end{abstract}

Neurokinin B (NKB) is a member of the tachykinin family of peptides, which are characterized by a common carboxylterminal region Phe-X-Gly-Leu-Met-NH2, and include substance $\mathrm{P}$, neurokinin $\mathrm{A}$, and NKB. NKB preferentially binds to neurokinin 3 receptor (NK-3R), encoded by the tachykinin receptor 3 gene which belongs to the superfamily of $G$ proteincoupled receptors $(1,2)$. NKB has recently emerged as a critical player in the central control of reproductive function (1). It has also been shown that the NKB/NK-3R system is

Correspondence to: Assoc. Prof. Tsuyoshi Shimo, Department of Oral and Maxillofacial Surgery, Okayama University Graduate School of Medicine, Dentistry and Pharmaceutical Sciences, 2-5-1 Shikata-cho, Kita-ku, Okayama 700-8525, Japan. Tel: +81 862356702, Fax: +81 862356704, e-mail: shimotsu@md.okayama-u.ac.jp

Key Words: Neurokinin 3 Receptor, Neurokinin B, oral squamous cell carcinoma. expressed in human, mouse, and rat uteri, and the expression and function of this system varies with age, menstrual cycle, and during the course of pregnancy $(3,4)$. In the pathological condition, NK-3R was expressed in human oral squamous cell carcinomas in head and neck tumors (5).

In this study, we statistically compared NK-3R expression in clinically-resected oral squamous cell carcinoma samples and investigated how NK-3R signaling involves tumorigenesis in tumor-induced bone destructive regions.

\section{Materials and Methods}

Immunohistochemical analysis of surgically resected samples. Surgically resected lower gingival, tongue, mouth floor, and buccal squamous cell carcinoma samples were collected as part of routine care by the authors at Okayama University Hospital (Okayama, Japan) in the years 2000-2013. No patient had received chemotherapy or radiation therapy before surgery. The retrospective study was approved by the Ethical Committee of the Okayama University Graduate School of Medicine, Dentistry and Pharmaceutical Sciences (Protocol No: 1949). Anti-NK-3R immunohistochemical analysis for NK-3R (anti-rabbit IgG, BIOSS, MA, USA) was performed as described previously (5). Thereafter, sections were viewed under a microscope, and the stained area was measured using imaging software (Lumina Vision, Mitani Corporation, Tokyo, Japan).

Cell lines and culture conditions. Human oral squamous cell carcinoma cell line HSC-2 was obtained from the RIKEN BioResource Center Cell Bank (Tsukuba, Japan). HSC-2 was incubated in Dulbecco's Modified Eagle Medium Nutrient Mixture F12 (DMEM-F12, Gibco, NY, USA) supplemented with $10 \%$ fetal bovine serum (Sigma-Aldrich, MO, USA) and $1 \%$ penicillin/ streptomycin (Gibco, NY, USA), and cultured in an atmosphere of $10 \% \mathrm{CO}_{2}$ at $37^{\circ} \mathrm{C}$.

Proliferation assay. HSC-2 cells were seeded on 6-well plates, and NKB $(0,0.01,0.1,1.0 \mu \mathrm{M}$, Peptide Institute Inc., Osaka, Japan) was added every $24 \mathrm{~h}$ for 1 week. Cells were collected and the number of cells was determined using a $\mathrm{TC} 10^{\mathrm{TM}}$ automated cell counter (Bio-Rad, Hercules, CA, USA). 

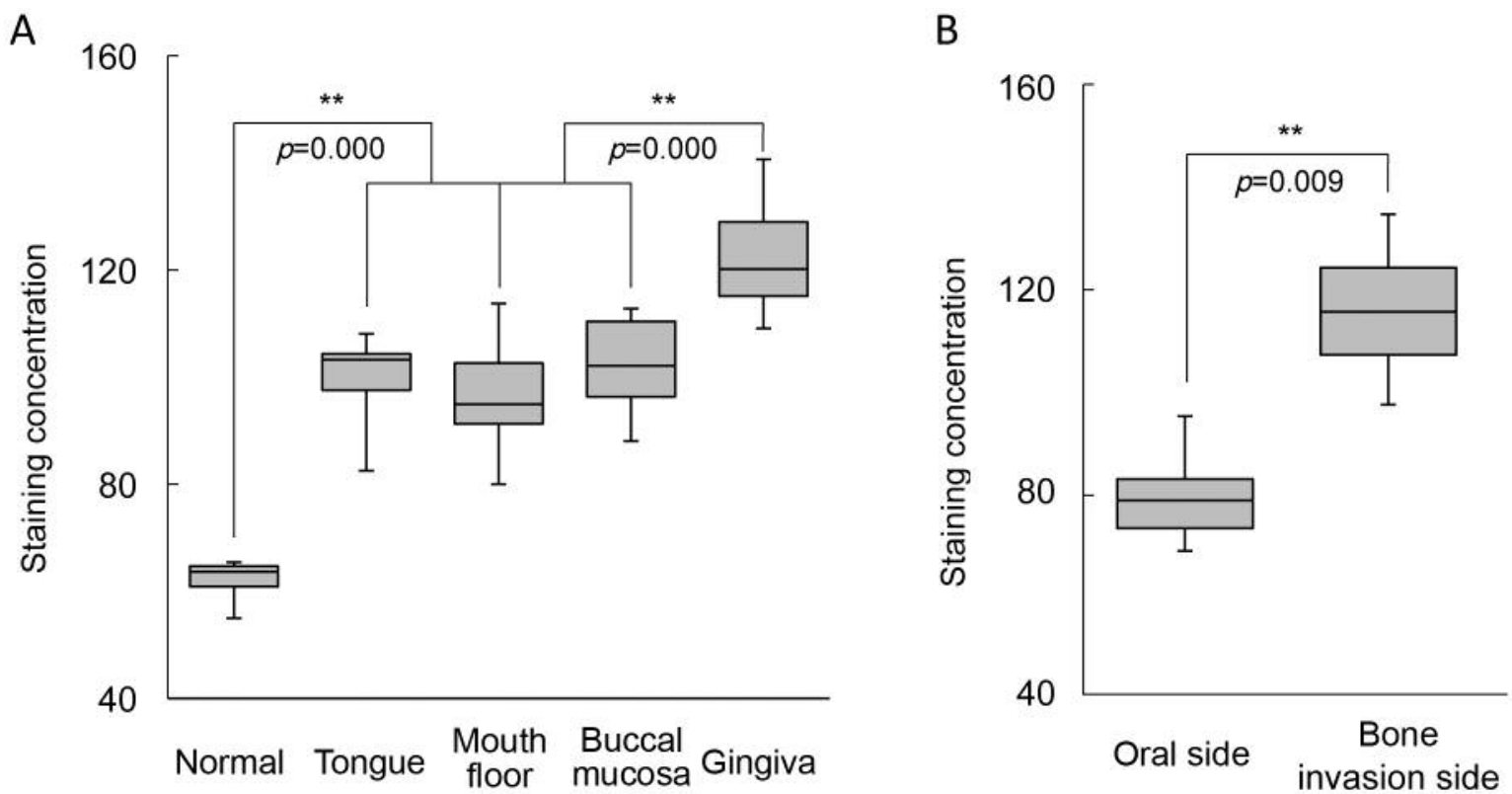

Figure 1. Comparison of the expression of $N K-3 R$ in normal epithelium and oral squamous cell carcinoma by site. A: Analysis of immunohistochemical staining concentration of $N K-3 R$ in normal gingiva and oral squamous cell carcinoma at the tongue, mouth floor, buccal mucosa, and gingiva. B: Comparison of immunohistochemical staining concentration of gingival oral squamous cell carcinoma at the oral side and bone invasion site $(n=8)$. Significant differences between the indicated groups were defined as $* p<0.05$, $* *<0.01$.

Scratch assay. HSC-2 cells were grown to subconfluence in a 6-well cell culture plate, and a single scratch was then made using the tip of a $200 \mu \mathrm{L}$ pipette. After washing with phosphate-buffered saline, complete medium containing NKB $(0,0.01,0.1,1.0 \mu \mathrm{M})$ was added. Serial photographs of the same scratched section were taken after 24 $\mathrm{h}$, and the amount of decrease in the scratched part was measured.

Invasion assay. HSC-2 invasion was assayed in a cell culture insert for a 24-well plate with a pore size of $8.0 \mu \mathrm{m}$ (Corning, NY, USA) according to the manufacturer's protocol. The upper wells were

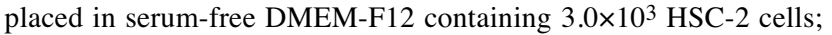
the lower wells were filled with DMEM-F12 containing NKB $(0$, $0.01,0.1,1.0 \mu \mathrm{M})$. After a $24-\mathrm{h}$ incubation of $10 \% \mathrm{CO}_{2}$ at $37^{\circ} \mathrm{C}$, the cells were stained with Diff-Quik (Sysmex Corp., Tokyo, Japan) and observed with a phase contrast microscope.

Real time-polymerase chain reaction (PCR) for NK-3R. HSC-2 cells were grown to subconfluence in a 6 -well cell culture plate, and NKB $(0,0.01,0.1,1.0 \mu \mathrm{M})$ was added. Total RNA was purified using the RNeasy Mini Kit (Qiagen, MD, USA) after $12 \mathrm{~h}$. Furthermore, cDNA was purified using an RNA PCR Kit (AMV) Ver. 3.0 (Takara Bio Inc., Shiga, Japan). The PCR was set up in a $10 \mu \mathrm{L}$ reaction volume containing $5 \mu \mathrm{M}$ SsoAdvanced Universal SYBR Green Supermix (BioRad, CA, USA), $10 \mu \mathrm{M}$ forward NK3R primer (5'-TTGCGGTGGACAGGTATATGG-3'), $10 \mu \mathrm{M}$ reverse NK-3R primer (5'-GGCCATTGCACAAAGCAGAG-3'), $2.2 \mu \mathrm{M}$ distilled water, and sample DNA. PCR was performed in a Chromo 4 Four-Color Realtime PCR System Brochure (BioRad, CA, USA) with an initial denaturation step at $95^{\circ} \mathrm{C}$ for $30 \mathrm{sec}$, followed by 35 cycles of $95^{\circ} \mathrm{C}$ for $5 \mathrm{sec}$ and $62^{\circ} \mathrm{C}$ for $30 \mathrm{sec}$.
Immunoblot analysis. HSC-2 cells were rinsed once with ice-cold phosphate buffered saline (PBS) and lysed in an ice-cold lysis buffer (50 mM Tris-HCl, pH 7.4, containing $150 \mathrm{mM} \mathrm{NaCl}, 1 \%$ Triton X-100, $1 \% \mathrm{NP}-40,10 \mathrm{mM} \mathrm{NaF}, 100 \mathrm{mM}$ leupeptin, $2 \mathrm{mg} / \mathrm{ml}$ aprotinin, and 1 $\mathrm{mM}$ phenylmethyl sulfonyl fluoride). Cell lysates containing $10 \mu \mathrm{g}$ of total protein in a lysis buffer were electrophoresed in Mini-PROTEAN TGX Gels (BioRad, CA, USA) and the proteins were transferred to Immobilon Transfer Membranes (Merck Millipore, Darmstadt, Germany). After blocking, the membranes were incubated with antiAkt rabbit monoclonal antibody (Cell Signaling Technology (CST), MA, USA), anti-phospho-Akt (p-Akt) rabbit monoclonal antibody (CST), anti-mammalian target of rapamycin (mTOR) rabbit polyclonal antibody (CST), anti-phospho-mTOR (p-mTOR) rabbit monoclonal antibody (CST) and anti- $\beta$-actin ( $\beta$-actin) rabbit monoclonal antibody (CST) at $4^{\circ} \mathrm{C}$ overnight. The secondary antibody used was anti-rabbit IgG, HRP-linked antibody (CST), and the antibodies were detected using EzWestLumi plus (ATTO, Tokyo, Japan).

Animal experiments. Mouse models of bone invasion by human oral squamous cell carcinoma were established in 4-week-old male BALB/c nude mice (CLEA Japan, Inc, Tokyo, Japan) by inoculating $6.2 \times 10^{5}$ HSC- 2 cells into the bone marrow space of the left tibial metaphysis. SB222200 (Sigma-Aldrich, St. Louis, MO, USA), which is a selective antagonist of the NK-3R, was intraperitoneally administered twice a week over a period of 4 weeks beginning 10 days after the inoculation. For a sham therapy as a control, dimethyl sulfoxide (Sigma-Aldrich) was intraperitoneally administered with the same protocol as SB222200. After 4 weeks, osteolytic bone destruction was assessed, and immunohistochemical analysis for NK-3R (BIOSS) and Ki-67 (anti-rabbit IgG, abcam, Cambridge, 

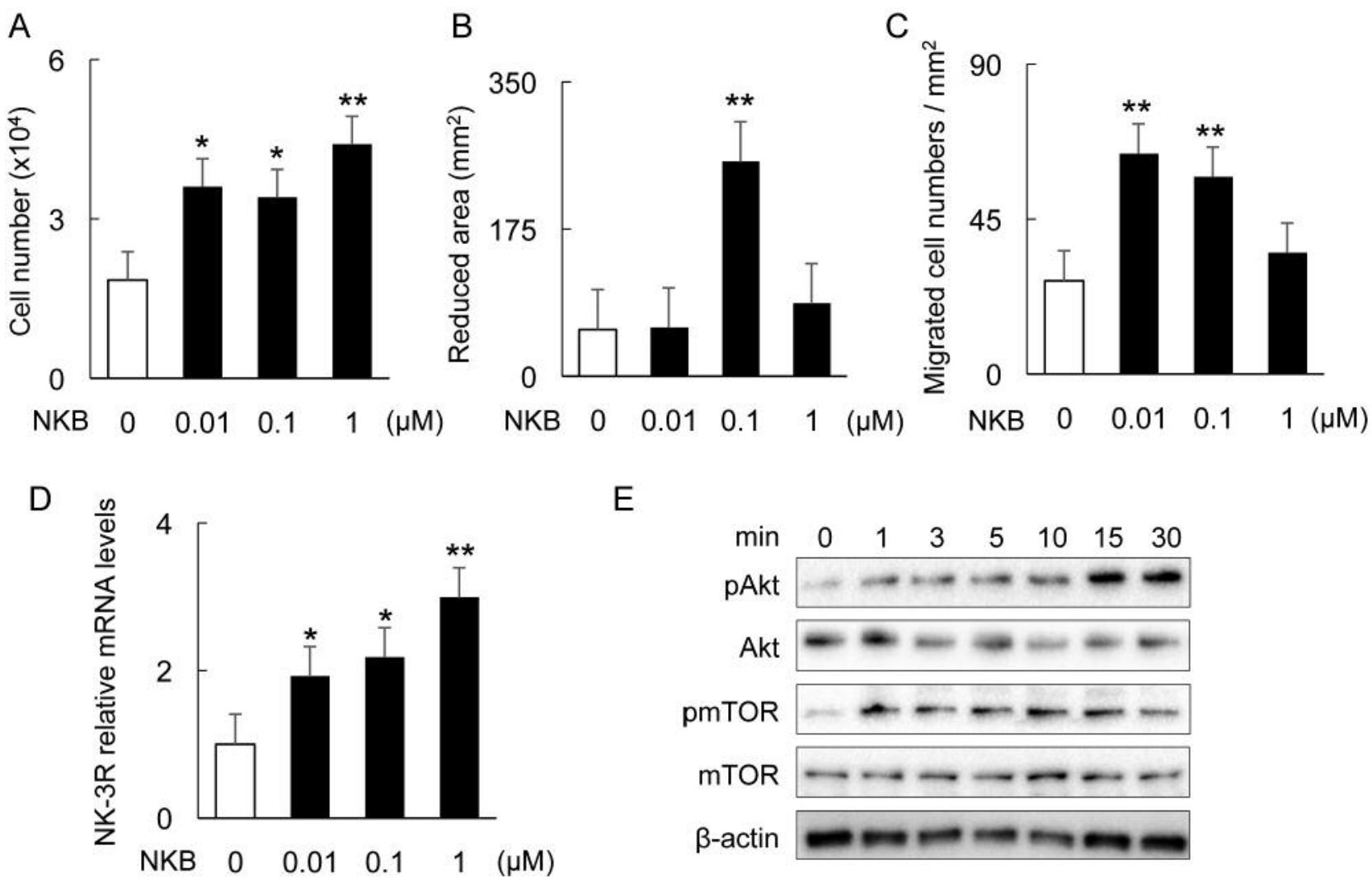

Figure 2. The effect of NKB on HSC-2 cell growth. A-C: The cell proliferation $(A)$, migration $(B)$, and chemotaxis $(C)$ were determined by the cell count, migration assay, and Boyden chamber methods after treatment with the indicated concentration of NKB. D: NK-3R expression by treatment with the indicated amount of NKB. E: Detection of anti-pAkt, Akt, pmTOR, and mTOR in HSC-2 cells by immunoblotting after treatment with $0.1 \mu M$ of NKB. Significant differences between the indicated groups were defined as $* p<0.05, * * p<0.01$.

UK) was performed as described previously (6). The protocols were approved by the Ethics Review Committee for Animal Experimentation of the Okayama University Graduate School of Medicine, Dentistry and Pharmaceutical Sciences (OKU-2016342).

Statistical analysis. Analysis of variance was used to immunohistochemically analyze clinical specimens. If there was overall significance, post hoc analysis was performed with Bonferroni's correction for multiple comparisons, simultaneously comparing all five parts: normal, tongue, mouth floor, buccal mucosa, and gingiva. We used unpaired Student's $t$-test for analysis of two groups. Differences were regarded as significant at a two-tailed $p<0.05$. The statistical software Excel and SPSS were used.

\section{Results}

Comparison of $N K-3 R$ expression in the surgically resected samples. In the pathological condition, NK-3R was expressed in the oral squamous cell carcinoma cells (5). To further investigate the expression of $\mathrm{NK}-3 \mathrm{R}$ in oral squamous cell carcinoma, we performed immunohistochemical analysis and quantified the strength of the expression. As shown in Figure 1A, NK-3R was significantly expressed in gingival squamous cell carcinoma compared to tongue, mouth floor, and buccal mucosa squamous cell carcinoma $(p<0.01)$. The normal gingival epithelium did not express NK-3R. Furthermore, more intense NK-3R expression was observed in tumor cells that had invaded the bone matrix compared to the oral side oral squamous cell carcinoma (Figure 1B) $(p<0.01)$.

The effect of $N K B$ on HSC-2 cell proliferation, migration, and chemotaxis. To investigate the effect of NKB on HSC-2 cells, we performed an in vitro experiment. As shown in Figure 2A, $0.01,0.1$, and $1.0 \mu \mathrm{M} \mathrm{NKB}$ significantly increased the proliferation of HSC- 2 cells. At a concentration of $0.1 \mu \mathrm{M}$, NKB significantly increased the migration of HSC-2 cells (Figure 2B), and at 0.01 and $0.1 \mu \mathrm{M}$ NKB significantly increased the chemotaxis of HSC-2 cells, as determined by Boyden chamber assay (Figure 2C). Stimulation of HSC-2 cells with $0.01,0.1$ and $1.0 \mu \mathrm{M}$ NKB resulted in a dosedependent increase of NK-3R mRNA (Figure 2D). To clarify whether the stimulation with NKB affected the proliferation signaling, the pAkt and pmTOR were analyzed by 
A
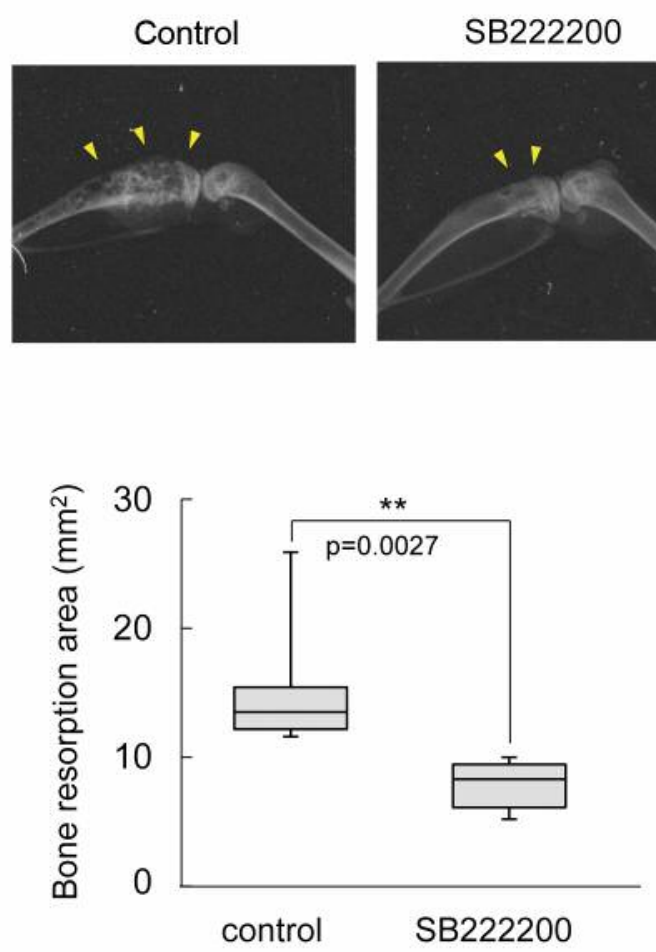

B

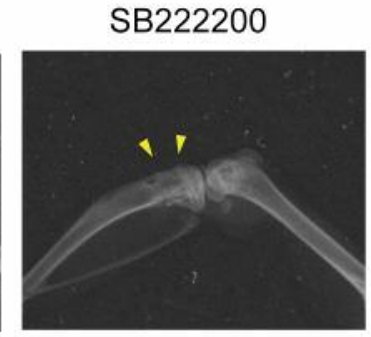

control
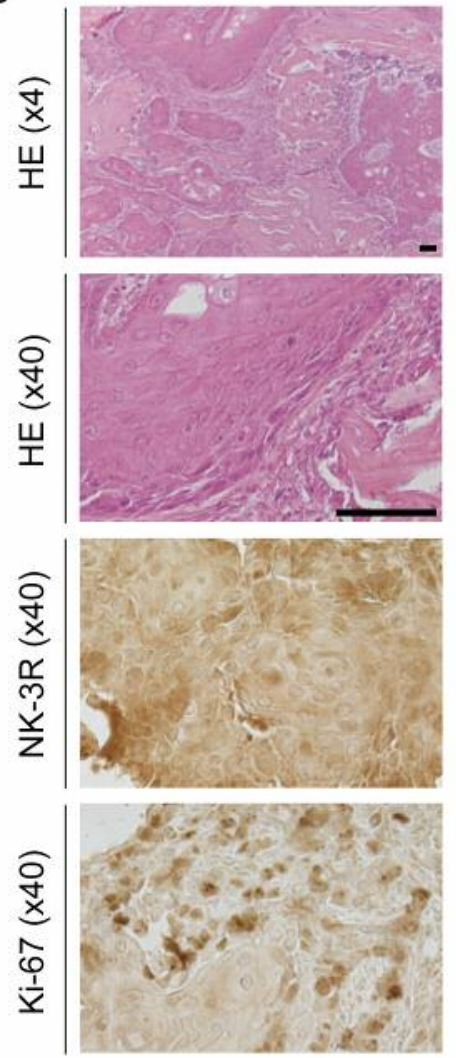

SB222200
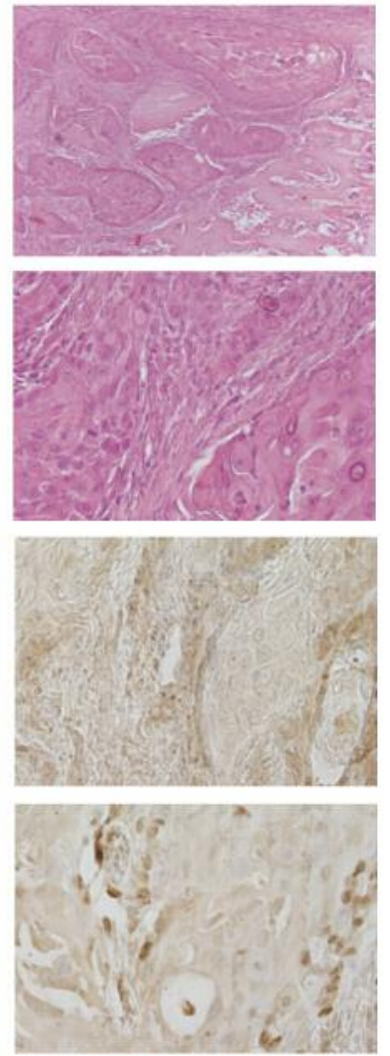

Figure 3. Radiographic and histologic analysis of tibial metaphysis-bearing HSC-2 cells in mouse models of bone invasion by human oral squamous cell carcinoma after treatment with NK-3R antagonist. A: Representative radiographs of the hind limb of the mouse model of bone invasion after $S B 222200$ or vehicle treatment. Arrowheads, osteolytic lesions. Osteolytic lesion area $\left(\mathrm{mm}^{2}\right)$ on the radiographs of the hind limbs treated with $S B 222200$ or vehicle ( $n=8 /$ group). The values represent the means \pm significant differences. Statistically significant differences $(* * p<0.01)$ between the indicated groups are marked by asterisks. B: Representative bone histology, NK-3R and Ki-67 expression in mouse models of oral squamous cell carcinoma bone invasion after SB222200 or vehicle treatment (Bar: $50 \mu \mathrm{m}$ ).

Immunoblot analysis. NKB increased the pAkt in a timedependent manner and it reached a maximum at $30 \mathrm{~min}$, while pmTOR reached a maximum after 1 min (Figure 2E).

NK-3R antagonist inhibits osteolytic bone destruction associated with invasion of oral squamous cell carcinoma. To clarify whether the inhibition of NKB signaling suppresses the osteolytic bone destruction induced by oral squamous cell carcinoma, we determined the inhibitory effect of NK-3R antagonist SB222200 on HSC-2 cell bone invasion. As shown in Figure 3A, the osteolytic lesions were clearly visible in the hind limb in the mouse models of bone invasion by human oral squamous cell carcinoma treated with vehicle. Few destructive lesions were detected in the hind limbs of the mouse group treated with SB222200. The total area of radiographic osteolytic lesions from all tibias was significantly suppressed by SB222200 treatment compared to the vehicle-treated control $(p<0.05)$. Histologic analysis revealed that the tumor cells invaded the bone marrow space and strongly expressed NK$3 \mathrm{R}$ and $\mathrm{Ki}-67$ in control mice. In contrast, NK-3R and Ki-67 expression in tumor cells after SB222200 treatment were significantly suppressed compared to the control (Figure 3B). These results suggest that SB222200 significantly suppressed oral squamous cell carcinoma tumorigenesis in the osteolytic bone destruction associated with oral squamous cell carcinoma invasion.

\section{Discussion}

Previous studies have implicated a role for NK-3R in oral squamous cell carcinoma (5). However, the expression levels of NK-3R and the role of NK-3R involvement in oral squamous cell carcinoma are not well understood. In the present study; the inhibition of NK-3R signaling with 
SB222200 inhibited the bone destruction associated with oral squamous cell carcinoma invasion by tumor proliferation and migration. These results indicated that NK-3R is a critical mediator of bone invasion in oral squamous cell carcinoma.

Here we have shown that the gingival oral squamous cell carcinoma invasion front is highly activated by NK-3R signaling. NKB at a concentration of $1 \mu \mathrm{M}$ stimulated HSC-2 cell proliferation and NK-3R mRNA expression, whereas HSC2 cell migration did not respond to $1 \mu \mathrm{M}$ NKB. The discrepancy in NKB concentration could be due to differences in the cell systems, and HSC-2 cells might select the cell function based on the NKB concentration in the tumor bone matrix. Previous studies involving histopathological examination have shown the importance of the expression of NKB in peripheral nerves in the mandible $(5,7)$. The data suggested that NKB was distributed in the mandibular bone matrix and bone marrow from the peripheral nerve spread around the jaw bone.

To investigate the molecular mechanism of antiosteoclastic effects, we examined the expression of NK-3R in tumor cells in a bone invasion model. Our data showed that the inhibition of NK-3R signaling down-regulated the NK-3R expression in HSC-2 cells. Our previous study showed that the NK-3R staining pattern was comparable to that of cytokeratin-17, a type 1 cytokeratin, and regulated mobility and migration in human oral squamous cell carcinoma resected samples. These data indicated that NKB signaling may be associated with the mobility and migration of oral squamous cell carcinoma cells in the bone matrix. However, the NK-3R signaling in cancer cells is not yet understood, whereas NK-3R seems to be regulated by estrogens at the central and peripheral levels (8). Estrogen receptor expression is strongly associated with cancer bone metastasis (9). Estrogen-related receptor alpha (ESRRA) has been shown to be up-regulated in oral squamous cell carcinoma, and ESRRA promotes the migration and invasion of the tumor (10). The Akt/mTOR signaling pathway is a key intercellular signaling system that drives cellular growth and survival, and hyperactivation of this pathway is implicated in the tumorigenesis of estrogenreceptor-positive breast cancer and in the resistance to endocrine therapy (11). These observations suggest that NK$3 \mathrm{R}$ expression may be up-regulated by ESRRA signaling and that NK-3R has a role in oral squamous cell carcinoma growth through the Akt/mTOR signaling pathway. Although the mechanism by which SB222200 suppressed the tumorigenesis of oral squamous cell carcinoma in the bone matrix is unknown, NKB up-regulated the pAkt/pmTOR signaling. Further studies are necessary in order to confirm the usefulness of the NK-3R targeted novel approach and whether SB222200 can regulate tumor growth, apoptosis, and Akt/mTOR signaling.

In summary, this study is, to the best our knowledge, the first to show that NK-3R signaling should be considered a target for the treatment of oral squamous cell carcinoma involved in bone destruction. These results suggest that regulation of NK-3R signaling alone or in combination with other agents might be considered a potential approach to treat advanced oral squamous cell carcinoma.

\section{Acknowledgements}

This work was supported by a Grant-in-Aid for Scientific Research (B) (\#JP26293428 to T.S.) from the Japan Society for the Promotion of Sciences.

\section{References}

1 Skorupskaite K, George JT, Veldhuis JD, Millar RP and Anderson RA: Interactions Between Neurokinin B and Kisspeptin in Mediating Estrogen Feedback in Healthy Women. J Clin Endocrinol Metab 101: 4628-4636, 2016.

2 Navarro VM: New insights into the control of pulsatile GnRH release: the role of Kiss1/neurokinin B neurons. Front Endocrinol 3: 1-9, 2012

3 Pennefather JN, Lecci A, Candenas ML, Patak E, Pinto FM and Maggi CA: Tachykinins and tachykinin receptors: a growing family. Life Sci 74: 1445-1463, 2004.

4 Kunimura Y, Iwata K, Ishigami A and Ozawa H: Age-related alterations in hypothalamic kisspeptin, neurokinin B, and dynorphin neurons and in pulsatile $\mathrm{LH}$ release in female and male rats. Neurobiol Aging 50: 30-38, 2017.

5 Obata K, Shimo T, Okui T, Matsumoto K, Takada H, Takabatake $\mathrm{K}$, Kunisada Y, Ibaragi S, Nagatsuka $\mathrm{H}$ and Sasaki A: Tachykinin Receptor 3 Distribution in Human Oral Squamous Cell Carcinoma. Anticancer Res 36: 6335-6341, 2016.

6 Okui T, Shimo T, Fukazawa T, Kurio N, Hassan NM, Honami T, Takaoka M, Naomoto Y and Sasaki A: Antitumor effect of temsirolimus against oral squamous cell carcinoma associated with bone destruction. Mol Cancer Ther 9: 2960-2969, 2010.

7 Ichiki T, Kuroishi KN, Gunjigake KK, Kobayashi S and Goto T: Neurokinin B activates the formation and bone resorption activity of rat osteoclasts. Neuropeptides 45: 239-244, 2011.

8 Navarro VM, Ruiz-Pino F, Sánchez-Garrido MA, García-Galiano D, Hobbs SJ, Manfredi-Lozano M, León S, Sangiao-Alvarellos S, Castellano JM, Clifton DK, Pinilla L, Steiner RA and Tena-Sempere M: Role of neurokinin B in the control of female puberty and its modulation by metabolic status. J Neurosci 32: 2388-2397, 2012.

9 Wei B, Wang J, Bourne P, Yang Q, Hicks D, Bu H and Tang P: Bone metastasis is strongly associated with estrogen receptorpositive/progesterone receptor-negative breast carcinomas. Hum Pathol 39: 1809-1815, 2008.

10 Tiwari A, Shivananda S, Gopinath KS and Kumar A: MicroRNA-125a reduces proliferation and invasion of oral squamous cell carcinoma cells by targeting estrogen-related receptor $\alpha$ : implications for cancer therapeutics. J Biol Chem 289: 32276-32290, 2014.

11 Ciruelos Gil EM: Targeting the PI3K/AKT/mTOR pathway in estrogen receptor-positive breast cancer. Cancer Treat Rev 40: $862-871,2014$

Received June 23, 2017

Revised July 17, 2017

Accepted July 18, 2017 